\title{
Eclipse de soleil du 28 juin 1908.
}

Voici les instants des contacts de cette éclipse partielle tels qu'ils ont été observés à l'observatoire de Genève:

Commencement de l'éclipse (T. M. Europe centrale).

M. Schaer $6^{\mathrm{h}} 16^{\mathrm{m}} 37^{\mathrm{s}} \quad$ M. Pidoux $6^{\mathrm{h}} 16^{\mathrm{m}} 50^{\mathrm{s}}$.

M. Schaer observait avec son réfracto-reffecteur de $35 \mathrm{~cm}$, diaphragmé à $20 \mathrm{~cm}$ et un grossissement de 100 fois. M. Pidoux observait avec le chercheur de l'équatorial Plantamour et un grossissement de 40.
Fin de l'éclipse (T. M. Europe centrale). M. Schaer à l'œil nue $\quad 7^{\mathrm{h}} 13^{\mathrm{m}} 20^{\mathrm{s}}$ > avec son instrument 7 I4 16 $\begin{array}{llll}\text { M. Gautier } & 7 & 14 & 35\end{array}$ M. Pidoux $\quad 7$ I 4 4 45 .

M. Gautier observait, par projection, avec une lunette de $80 \mathrm{~mm}$ d'ouverture. - M. Pidoux observait avec l'équatorial Plantamour de $28 \mathrm{~cm}$ et un grossissement de 200 .

Observatoire de Genève, 1908 Octobre.

R. Gautier.

\section{Notice regardant le terme $\mathrm{z}$ dans la variation de la latitude.}

Dans son savant article,$O n$ the results of the international latitude observations (Astr. Nachr. 428r). Monsieur Hirayama, entre autres choses, est arrivé a la conclusion suivante :

The amplitude of $z$-variation increases as the mean zenith distance (within the limits $0^{\circ}-20^{\circ}$ ) $\ldots$

Torino, le 12 novembre 1908 .
Or, je tiens à rappeler que dès I 900 j'ai publié une remarque identique: $1^{\circ}$ dans les Comptes rendus de l'Académie des Sciences, 5 février $1900 ; 2^{\circ}$ dans le Bulletin Astronomique, avril 1900; $3^{\circ}$ dans les Pubblicazioni dell' Osservatorio privato di Collurania No. 2 I 900.

Fean Boccardi.

\section{Notiz betr. ST Ophiuchi.}

Bezliglich des Veränderlichen ST Ophiuchi (A. N. 4284) möchte ich zu der von Herrn Guthnick gegebenen Erklärung bemerken, daß die Kurve trotz des relativ langen horizontalen Stückes doch merklich genug an die Kurven von $\delta$ Cephei und $\eta$ Aquilae erinnert, um eine ähnliche Ursache des Lichtwechsels, also wohl das Bestehen eines engen Sternpaares glaubhaft zu machen. Besonders das zweite Maximum erinnert an jene Sterne.

Münster, 1908 Nov. 29.

F. Plassmann.

\section{Notiz betr. (653) [1907 BK].}

Von dem Planeten (653) [1907 BK] (vergl. A. N. r79.95 und I15) kann ich noch eine Opposition nachweisen;

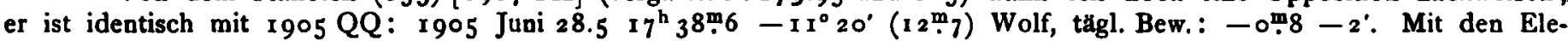

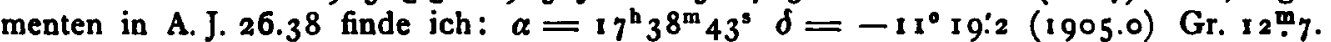

Tempelhof, 1908 Nov. 5 .

A. Berberich.

\section{Zur Geschichte der astronomischen MeBwerkzeuge.}

Dr. Repsold beabsichtigt, seine obengenannte Arbeit bis gegen das Ende des verflossenen Jahrhunderts fortzufuhren. Er bittet die Direktoren aller Sternwarten und geodätischen Institute, ihm dabei behilflich zu sein, indem sie ihm freundlichst diejenigen Teile der aus ihren Anstalten hervor.

gegangenen Publikationen, welche Beschreibungen und Abbildungen astronomischer Instrumente enthalten, zur Verfügung stellen. Dr. Repsold würde sehr dankbar sein für eine baldige Zusendung unter der Adresse Hamburg, Claus Groth-Straße 96.

(28) Bellona. Korrektion der Ephemeride (B. J. 1910): 1908 Nov. $28-17^{3} 77$ +0 9"0. W. Luther. (352) Gisela. 1908 Nov. $2613^{\mathrm{h}} 49^{\mathrm{m}} 5^{\mathrm{s}}$ Kopenhagen $\alpha$ app. $=4^{\mathrm{h}} 50^{\mathrm{m}} 5^{2} \cdot 35(9.091) \delta$ app. $=+22^{\circ} 16^{\prime} 20^{\mathrm{m}} \cdot 5$ (0.693). C. F. Pechiule.

Inhalt zu Nr. 4287. Th. Albrecht. Provisorische Resultate des Internationalen Breitendienstes auf dem Sudparallel in der Zeit von I go6.4-I908.4 229. - A. Kopff. Ein merkwürdiges Meteor vom 23. Oktober 1908. 237. $-R$. Gautier. Changements d'aspect de la comète Morehouse $(1908 \mathrm{c}$ ). 237. $-W$. Winkler. Beobachtungen des Kometen $1908 \mathrm{c}$ (Morehouse). 239. $-R$. Farry-Desloges. Sur un nouvel anneau de Saturne. 239. - P. Lowell. The spectra of the major planets. 24I. $-M$. Wolf. Photographische Aufnahmen von kleinen Planeten. 24I. - H. H. Kritainger. Kreisbahnelemente und Ephemeride für den Planeten I 908 EP. 241. - R. Gautier. Eclipse de soleil du 28 juin 1908. $243-\mathcal{F}$. Boccardi. Notice regardant le terme 8 dans la variation de la latitude. 243. - F. Plassmann. Notiz betr. ST Ophiuchi. 243. - A. Berberich. Notiz betr. (653) [1907 BK]. 243. Zur Geschichte der astronomischen Meßwerkzeuge. 243. - Kleine Mitteilungen. 243. 